Beiträge zu

Kriminologie und Strafrecht

Band 2 


\section{Susanne Henninger}

\section{Nichtdeutsche Beschuldigte im Jugendstrafverfahren}

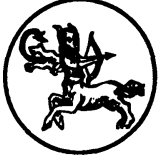

Centaurus Verlag \& Media UG 2003 
Susanne Henninger, geb. 1970, studierte Rechtswissenschaften in Straflburg (Frankreich) und Heidelberg. Sie promovierte 2001 mit dieser Arbeit an der Universität Heidelberg und ist derzeit juristische Referentin im Landeskirchenamt der Evangelisch-Lutherischen Kirche in Bayern.

\section{Die Deutsche Bibliothek - CIP-Einheitsaufnahme}

Bibliographische Information der Deutschen Bibliothek Die Deutsche Bibliothek verzeichnet diese Publikation in der Deutschen Nationalbibliographie; detaillierte bibliographische Daten sind im Internet über http://dnb.ddb.de abrufbar.

ISBN 978-3-8255-0386-4 ISBN 978-3-86226-440-7 (eBook)

DOI 10.1007/978-3-86226-440-7

\section{ISSN 1610-9538}

Alle Rechte, insbesondere das Recht der Vervielfältigung und Verbreitung sowie der Übersetzung, vorbehalten. Kein Teil des Werkes darf in irgendeiner Form (durch Fotokopie, Mikrofilm oder ein anderes Verfahren) ohne schriftliche Genehmigung des Verlages reproduziert oder unter Verwendung elektronischer Systeme verarbeitet, vervielfältigt oder verbreitet werden.

(C) CENTAURUS-Verlags GmbH \& Co. KG, Herbolzheim 2003

Satz: typo \& text Gabi Mang Umschlaggestaltung: DTP-STUDIO, Antje Walter, Hinterzarten 


\section{Inhalt}

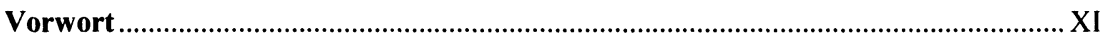

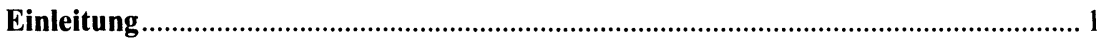

\section{Teil: Lebenssituation und Kriminalitätsbelastung von Ausländern}

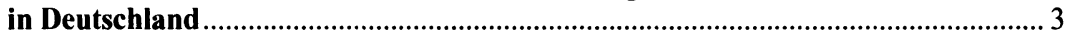

1. Kapitel: Die Lebenssituation der (jungen) Ausländer in Deutschland ...................................3

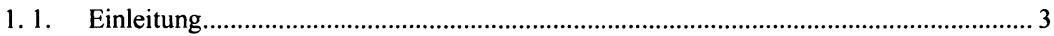

1. 2. Überblick über die Entwicklung der ausländischen Bevölkerung in Deutschland ...........4

1. 3. Nationalitätsstruktur...........................................................................................9

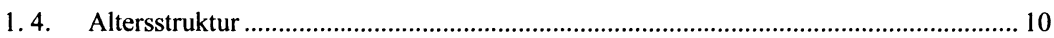

1. 5. Geschlechtsstruktur......................................................................................... 10

1. 6. Lebenslage der jungen Nichtdeutschen...................................................................... 11

1. 6. 1. Identität, Biographie und Familie................................................................ 12

1. 6. 2. Schulische und berufliche Situation ............................................................... 20

1. 6.3. Freizeitkontakte und ethnische Selbstidentifikation........................................ 23

1. 6. 4. Aufenthaltsdauer und Rückkehrperspektive.................................................. 26

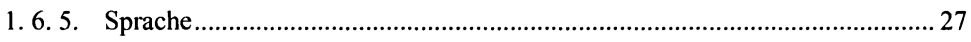

2. Kapitel: Die Belastung der Ausländer nach der PKS ……………………………............28

2. 1. Zur Kategorie „Ausländerkriminalität“ ..................................................................... 28

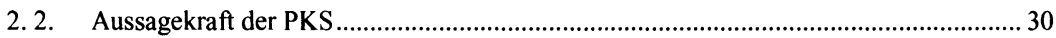

2. 3. Belastung der Ausländer nach der PKS (1999) ........................................................... 34

2. 3. 1. Deliktsschwerpunkte ............................................................................... 34

2. 3. 2. Aufgliederung nach Nationalitäten (Bundesgebiet insgesamt) ........................ 35

2. 3. 3. Aufgliederung nach dem Aufenthaltsgrund (gesamtes Bundesgebiet)............. 35

2. 3. 4. Alters- und Geschlechtsstruktur der nichtdeutschen Tatverdächtigen (gesamtes Bundesgebiet) ................................................................................ 36 
3. Kapitel: Die Kriminalitätsbelastung der Nichtdeutschen, insbesondere der jungen Nichtdeutschen, anhand der empirischen Forschung .............................................38

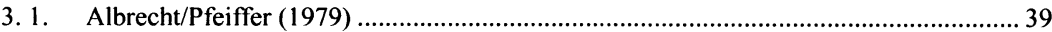

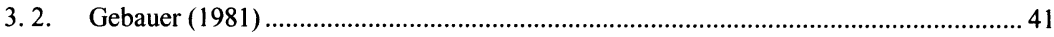

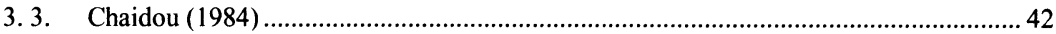

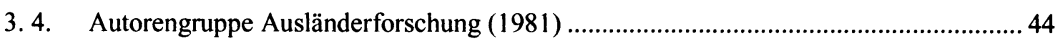

3. 5. Landeskriminalamt Baden-Württemberg.................................................................... 48

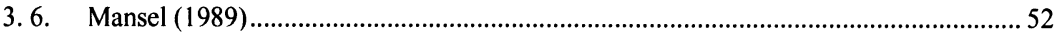

3. 7. Rodel (1976), Pitsela (1986) und Piehler (1991) …............................................................ 54

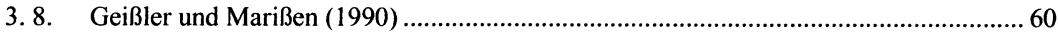

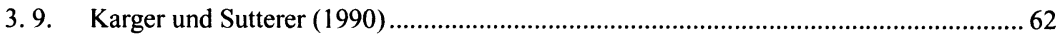

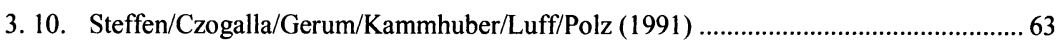

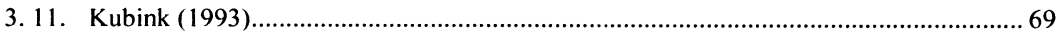

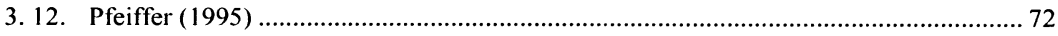

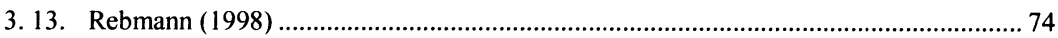

3. 14. Pfeiffer/Delzer/Enzmann und Wetzels (1998) ........................................................... 77

4. Kapitel: Erklärungsansätze zur Kriminalitätsentstehung bei Ausländern..........................78

4. 1. Kulturkonflikttheorie und ihre Modifikationen ............................................................... 79

4. 2. Anomietheorie, soziale Mangellage und eingeschränkte Zugangschancen .................... 82

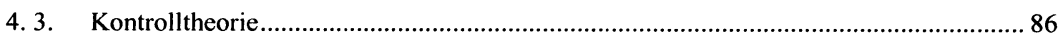

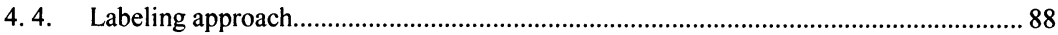

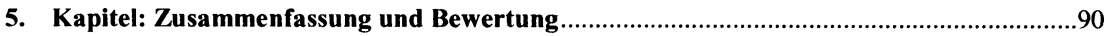

2. Teil: Der Verlauf des Jugendstrafverfahrens....................................................... 95

6. Kapitel: Junge nichtdeutsche Beschuldigte : Verlauf des strafrechtlichen Selektionsprozesses zwischen Anzeigenerstattung und gerichtlicher Entscheidung...........95

6. 1. Einleitung - Der strafrechtliche Selektionsprozeß ........................................................ 95

6. 2. Der Schwund zwischen Tatverdächtigen und Abgeurteilten- $/$ Verurteiltenzahlen bei den Nichtdeutschen

6. 3. Empirisch-statistische Befunde zum Aburteilungs/Verurteilungsrisiko von jungen Deutschen und Nichtdeutschen .98

6. 3. 1. Untersuchung von Mansel (1989) ................................................................ 98

6. 3. 2. Untersuchung von Geißler/Marißen (1990) .................................................. 99

6. 3. 3. Untersuchung von Pfeiffer/Schöckel (1990) .............................................. 100

6. 3. 4. Untersuchung von Reichertz, Donk und Schröer ......................................... 101

6. 4. Die „Reduktion des Tatvorwurfs“: ein Phänomen vergangener Jahre? ........................ 101

6. 4. 1. Untersuchung von Steffen (1996) ............................................................. 102

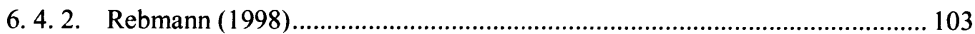

6. 5. Erklärungsansätze für die „Reduktion des Tatvorwurfes“............................................ 104 
6. 5. 1. Das selektive Anzeigeverhalten der Bevölkerung......................................... 104

6. 5. 2. Die Anzeigebereitschaft innerhalb derselben ethnischen Gruppe ................ 109

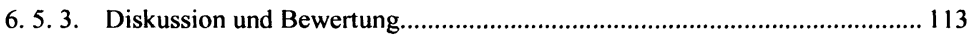

6. 5. 4. Die polizeiliche Arbeit und ihre Bedeutung für den strafrechtlichen Selektionsproze $\beta$...................................................................................... 115

6. 5. 4. 1. Die Polizei in einer ,multikulturellen“ Gesellschaft -

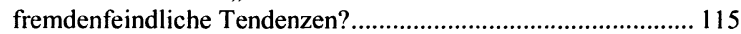

6. 5. 4. 1. 1. Studie von Jaschke (1997) ......................................................... 117

6. 5. 4. 1. 2. PFA-Studie von Bornewasser u. a. (1996) ................................ 118

6. 5. 4. 1. 3. Parlamentarischer Untersuchungsausschuß „Hamburger Polizei“ ................................................................ 121

6. 5. 4. 1. 4. Mletzko und Weins (1999) ...................................................... 121

6. 5. 4. 2. Selektives Überwachungsverhalten der Polizei .......................... 123

6. 5. 4. 3. Selektive Arbeit der Polizei ....................................................... 125

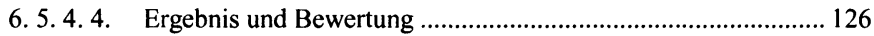

6. 5. 4. 5. Die Polizei in der Wahrnehmung ausländischer Jugendlicher... 128

6. 5. 5. Die Reduktion des Tatvorwurfs als Folge polizeilicher Ermittlungspraxis... 132

6. 5. 5. 1. Die Vernehmung ausländischer deutschsprachiger Beschuldigter - das Phänomen der informellen Aussageverweigerung ...... 133

6. 5. 5. 2. Die Vernehmung nichtdeutschsprachiger Beschuldigter mittels Dolmetscher.................................................................. 136

6. 5. 5. 3. Ergebnis und Bewertung ........................................................... 139

6. 5. 6. Exkurs: Ermittlungsprobleme und Besonderheiten im Umgang mit jungen nichtdeutschen Beschuldigten ...................................................... 139

6. 5. 7. Die Rolle der Staatsanwaltschaft im strafrechtlichen Selektionsprozeß ....... 144

6. 5. 7. 1. Einstellungspraxis der Staatsanwaltschaft gegenüber jungen Nichtdeutschen anhand empirischer Befunde................ 145

6. 5. 7. 2. $§ 154$ b StPO - eine ,ausländerspezifische“"Möglichkeit der Verfahrenseinstellung....................................................... 148

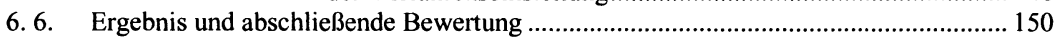

7. Kapitel: Das Problem der Altersfeststellung bei jungen ausländischen Tatverdächtigen.

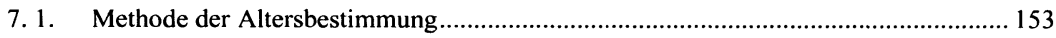

7. 2. Rechtsgrundlage der röntgenologischen Untersuchung und deren Tauglichkeit für die Altersbestimmung bei jungen Nichtdeutschen.. 154

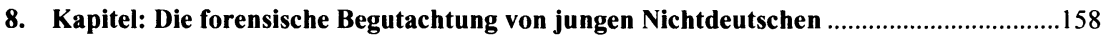

9. Kapitel: Die Frage der Verantwortlichkeit gemäß § 3 JGG und die Reifeentscheidung gemäß § 105 JGG .161

9. 1. Strafrechtliche Verantwortlichkeit bei nichtdeutschen Jugendlichen .......................... 161

9. 2. Die Reifeentscheidung gemäß § 105 JGG .............................................................. 162

9. 3. Kulturabhängigkeit testpsychologischer Untersuchungen als Problem bei der Beurteilung der geistigen Reife 167 
10. Kapitel: Verhängung von Untersuchungshaft bei ausländischen Jugendlichen und Heranwachsenden.

10. 1. Rechtliche Grundlagen der Verhängung von Untersuchungshaft gegenüber Jugendlichen und Heranwachsenden 169

10. 2. Quantitative Entwicklung der U-Haft bei Jugendlichen und Heranwachsenden 174

10. 3. Die Verhängung von U-Haft gegenüber ausländischen Jugendlichen und Heranwachsenden 178

10. 3. 1. Der Haftgrund der Fluchtgefahr und die Ausländereigenschaft. 189

10. 3. 2. Apokryphe Haftgründe. 196

10. 3. 3. Haftentscheidungshilfe durch die JGH und U-Haft-Vermeidung bei ausländischen Jugendlichen. 201

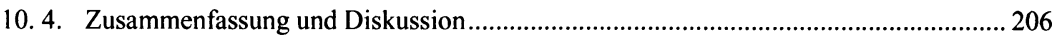

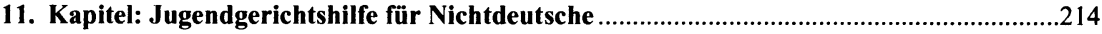

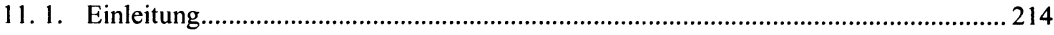

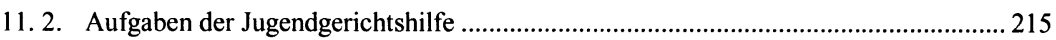

11. 3. Mitwirkung der JGH im Verfahren gegen nichtdeutsche Jugendliche und Heranwachsende und ihre Grenzen........................................................................ 217

11. 3. 1. Beteiligung der JGH im Verfahren gegen ausländische Beschuldigte.......... 217

11. 3. 2. Der Umgang der JGH mit ausländischen Jugendlichen und Heranwachsenden 218

11. 3. 3. Die JGH in der Wahrnehmung junger Nichtdeutscher.................................. 224

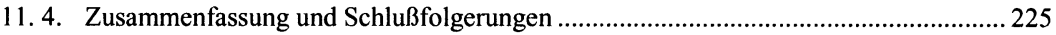

12. Kapitel: Junge nichtdeutsche Beschuldigte in der gerichtlichen Verhandlung ................227

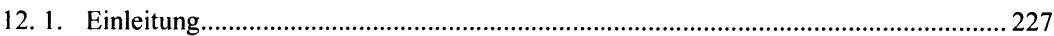

12. 2. Die Kommunikations- und Interaktionssituation vor Gericht ..................................... 228

12. 3. Kommunikations- und Interaktionsprobleme in der Gerichtsverhandlung mit jungen ausländischen Beschuldigten

12. 3. 1. Kulturelle Bezugsrahmen ..................................................................... 231

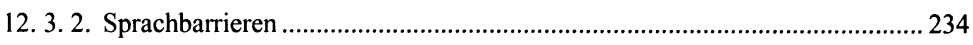

12. 3. 3. Gestaltung interkultureller Kommunikation vor Gericht .............................. 235

13. Kapitel: Die notwendige Verteidigung von Nichtdeutschen im Jugendstrafverfahren

13. 1. $§ 68$ Nr.1 JGG iVm § 140 Abs.2 StPO als Problemschwerpunkt ................................. 238

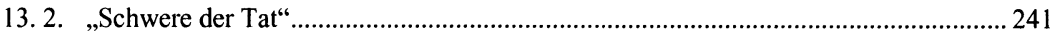

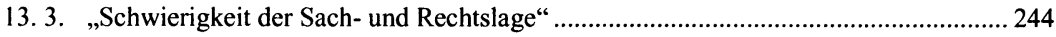

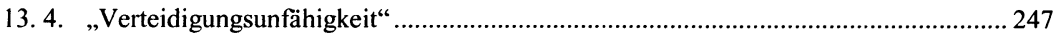

13. 5. „Sprachunkundigkeit“: eine ausreichende Begründung für eine notwendige Verteidigung gemäß $\S 140$ Abs.2 StPO in der Rechtsprechung? 249

13. 6. Zusammenfassung und Folgerungen 
14. Kapitel: Die Beteiligung von Dolmetschern im Strafverfahren gegen ausländische Beschuldigte

14. 1. Deutsch als Gerichtssprache. Die Regelung der $\S \S 184,185$ GVG, ihre verfassungsrechtliche Grundlagen und ihr Anwendungsbereich

14. 2. Umfang der Übersetzung

14. 2. 1. Verhandlung unter Beteiligung von Personen, die der deutschen Sprache nicht mächtig sind

14. 2. 2. Schriftliche Entscheidungen und Erklärungen des Gerichts 261

14. 2. 3. Erklärungen der Prozeßbeteiligten 264

14. 3. Art und Weise des Übersetzens in der gerichtlichen Verhandlung...............................267

14. 4. Dolmetscher: Sprachmittler oder Mittler zwischen zwei Kulturen? ............................ 271

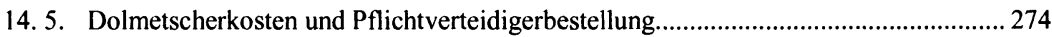

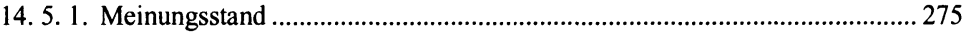

14. 5. 1. 1. Unentgeltlichkeit im Sinne des Art. 6 Abs. 3 lit. e EMRK........ 275

14. 5. 1. 2. Anspruch auf Beiziehung eines Dolmetschers

zur Verständigung in vorbereitenden Gesprächen..................... 276

14. 5. 1. 3. Kostentragung....................................................................... 278

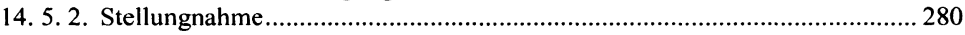

14. 6. Bedeutung der Dolmetscherauswahl - Schlußfolgerungen aus der

Kommunikationssituation und der Dolmetscherproblematik...................................... 282

15. Kapitel: Die jugendgerichtliche Sanktionierung bei ausländischen Jugendlichen ............286

15. 1. Bedeutung der ,Ausländereigenschaft“ im Rahmen der Strafzumessung .................... 287

15. 2. Gerichtliche Sanktionierung von ausländischen Jugendlichen

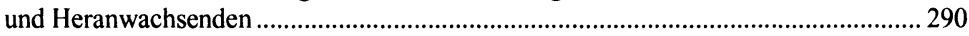

15. 3. Zusammenfassung und Diskussion der Ergebnisse .................................................... 294

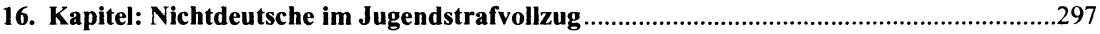

16. 1. Einleitung - Die ethnische Zusammensetzung im Jugendstrafvollzug.......................... 297

16. 2. Rechtsgrundlagen und Ausgestaltung des Jugendstrafvollzuges .................................. 300

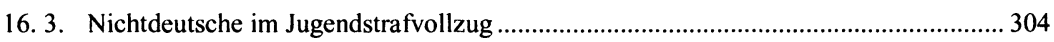

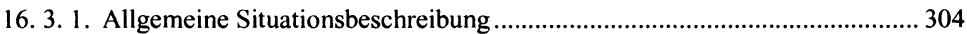

16. 3. 2. Einfluß des Ausländerrechts auf den Strafvollzug, insbesondere die Gewährung von Vollzugslockerungen nach den VVJug........................... 309

16. 3. 3. Ergebnisse empirischer Studien zum (Jugend-) Strafvollzug......................... 314

16. 4. Das Absehen von der Strafvollstreckung gemäß § 456a StPO ................................... 332

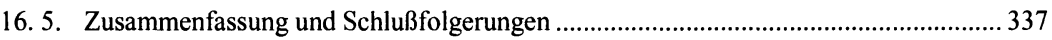


3. Teil: Ausländerrecht und Jugendstrafrecht: Die (Un-) Vereinbarkeit von Gefahrenabwehr und Erziehungsgedanken

17. Kapitel: Ausländerrechtliche Konsequenzen von Straffälligkeit - insbesondere die Ausweisung

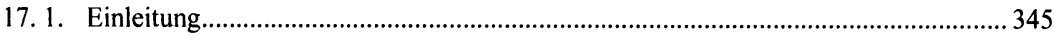

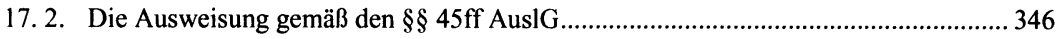

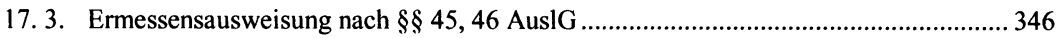

17. 4. Spezial- und Generalprävention als Motive der Ausweisung wegen

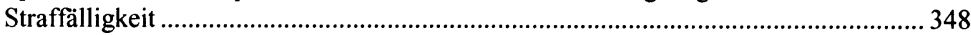

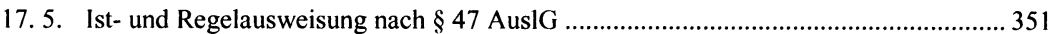

17. 6. Besonderer Ausweisungsschutz für minderjährige und heranwachsende Ausländer.

17. 7. Junge Ausländer im Spannungsfeld zwischen Jugendstrafrecht und Ausländerrecht

17. 7. 1. Wechselwirkung von (Jugend-)Strafrecht und Ausländerrecht

17. 7. 2. Erziehungsgedanke des Jugendstrafrechts contra ausländerrechtliche Gefahrenabwehr - Unvereinbarkeit jugendstrafrechtlicher und ausländerrechtlicher Zielsetzung.

17. 7. 3. Ausweisung als strafrechtliche Nebenfolge, Nebenstrafe oder entsprechend der $\S \S 69 \mathrm{ff}$ StGB?

17. 8 . Schlußfolgerungen 369

4. Teil: Schlußbetrachtung 371

Literaturverzeichnis 


\section{Vorwort}

Die vorliegende Arbeit wurde im Wintersemester 2001/2002 von der Juristischen Fakultät als Dissertation angenommen.

Bedanken möchte ich mich bei dem Betreuer dieser Arbeit, Professor Dr. Dieter Dölling vom Institut für Kriminologie in Heidelberg, für die Anregung zur Beschäftigung mit diesem Thema und seine langjährige Unterstützung sowie bei Professor Dr. Thomas Hillenkamp für die Erstellung des Zweitgutachtens.

Diese Arbeit wäre nicht möglich gewesen ohne die großherzige und geduldige Unterstützung durch Eltern und Freunde sowie die aufbauende und kritische Begleitung durch die Mitarbeiter am Institut für Kriminologie. Sie alle haben zum Gelingen dieser Arbeit beigetragen und dafür möchte ich ihnen von Herzen danken.

München, im Juli 2002

Susanne Henninger 